\title{
Different Activity of the Biological Axis VEGF-Flt-1 (fms-Like Tyrosine Kinase 1) and CXC Chemokines between Pulmonary Sarcoidosis and Idiopathic Pulmonary Fibrosis: A Bronchoalveolar Lavage Study
}

\author{
Katerina M. Antoniou, ${ }^{1}$ Giannoula Soufla, ${ }^{2}$ Athanasia Proklou, ${ }^{1}$ George Margaritopoulos, ${ }^{1}$ \\ Christiana Choulaki, ${ }^{3}$ Rena Lymbouridou, ${ }^{2}$ Katerina D. Samara, ${ }^{1}$ Demetrios A. Spandidos, ${ }^{2}$ \\ and Nikolaos M. Siafakas ${ }^{1}$ \\ ${ }^{1}$ Department of Thoracic Medicine, University Hospital, Medical School, University of Crete, Heraklion, 71110 Crete, Greece \\ ${ }^{2}$ Laboratory of Virology, Medical School, University of Crete, Heraklion, Greece \\ ${ }^{3}$ Department of Rheumatology, Clinical Immunology and Allergy, University Hospital, University of Crete, Heraklion, Greece
}

Correspondence should be addressed to Katerina M. Antoniou, katerinaantoniou@yahoo.gr

Received 15 July 2009; Revised 27 September 2009; Accepted 7 December 2009

Recommended by C. Pauza

\begin{abstract}
Background. We have previously shown a different local and systemic angiogenic profile of CXC chemokines in Idiopathic Pulmonary Fibrosis (IPF) patients compared to sarcoidosis. In particular, sarcoidosis showed an angiostatic microenvironment, as compared with the angiogenic cytokine milieu seen in IPF. Purpose of the Study. Our aim was to further investigate the aforementioned finding by measuring the expression of different chemokines in granulomatous and fibrotic diseases. We estimated the levels of vascular endothelial growth factor (VEGF) and its high-affinity receptor, Flt-1 (fms-like tyrosine kinase 1), in bronchoalveolar lavage fluid (BALF) of patients with IPF and pulmonary sarcoidosis. We have also investigated the mRNA expression of angiogenetic chemokines' receptors such as CXCR2 and CXCR3 and the biological axis of stromal derived factor- $1 \alpha$ (SDF-1 $\alpha$ or CXCL12 $\alpha /$ CXCL12 $\beta$ ) and receptor, CXCR4. Methods. We studied prospectively three groups of patients: (i) one group of 18 patients with IPF, (ii) one group of 16 patients with sarcoidosis, and (iii) 10 normal subjects. Results. A statistically significant increase has been detected in VEGF mRNA expression in IPF in comparison with pulmonary sarcoidosis $(P=.03)$. In addition, a significant increase has been measured in CXCL12 $\alpha$ in sarcoidosis in comparison to IPF $(P=.02)$. Moreover, a statistically significant decrease has been found in Flt-1 protein levels in pulmonary sarcoidosis in comparison with IPF $(P=.03)$. A significant increase in VEGF $(P=.03)$ and CXCR4 $(P=.03)$ mRNA levels has been also detected in sarcoidosis' patients when compared with healthy controls. Conclusions. Our data suggest that increased expression of Flt- 1 and downregulation of CXCL12 in IPF may further support the hypothesis of a different angiogenetic profile between fibrotic and granulomatous diseases. However, further studies are needed in order to better investigate these enigmatic diseases.
\end{abstract}

Copyright (C) 2009 Katerina M. Antoniou et al. This is an open access article distributed under the Creative Commons Attribution License, which permits unrestricted use, distribution, and reproduction in any medium, provided the original work is properly cited.

\section{Introduction}

Angiogenesis has been implicated in the pathogenesis of several fibrotic lung conditions, including idiopathic pulmonary fibrosis (IPF). This process plays a significant role in wound healing and contributes to the fibroproliferation and extracellular matrix deposition [1]. Neovascularization in fibroproliferative disorders is regulated by an opposing balance between angiogenic and angiostatic factors $[2,3]$.

Sarcoidosis continues to be a disease of research interest because of its complicated immune mechanisms and elusive etiology [4]. So far, it has been established that granulomatous inflammation in sarcoidosis is predominately a Thelper 1 immune response mediated by a complex network of lymphocytes, macrophages, and cytokines [5]. The cause 
of progression to a chronic and potentially fibrotic form is still unclear and up to $30 \%$ of patients have chronic course of the lung disease, resulting in progressive loss of lung function $[6,7]$.

Recently the chemokine receptors expressed specifically on Th1 cells were identified and it was reported that Th1 cells are characterized by the expression of CCR5 or CXC chemokine receptor 3 [8]. While CXCR3/CXCR3 ligands inhibit angiogenesis, CXCR3 ligands play a pivotal role in orchestrating Th1 cytokine-induced cell-mediated immunity via the recruitment of mononuclear and CD4+ T-cells expressing CXCR3 and consequently via the granuloma formation $[4,5]$. So far, there are only few studies in the literature implicating angiogenesis in the immunomodulatory cascade of sarcoidosis. BAL lymphocytes in sarcoidosis have been reported to be highly positive for CCR5 and CXCR3 $[9,10]$. Known ligands for CXCR3 are three angiostatic ELR $^{-}$CXC chemokines (Mig/CXCL9, IP-10/CXCL10, and ITAC/CXCL11) [9-12]. Recent studies have shown that these chemokines play an important role in the accumulation of Th1 lymphocytes in sarcoid lungs [11]. We have shown that sarcoidosis exhibits a distinct angiostatic profile, as shown by an $\mathrm{ELR}^{-} \mathrm{CXC}$ chemokine upregulation in comparison to IPF patients [12]. On the other hand, ELR+ CXC chemokines (IL-8, ENA-78, and GRO- $\alpha$ ) sharing the same receptor, CXCR2, were found increased in IPF [12], while a downregulation of their levels was recently shown after treatment [13].

Vascular endothelial growth factor (VEGF) is a potent growth factor for endothelial cells that regulates vascular permeability and the stimulation of angiogenesis. VEGF is crucial in lung development and maintenance during the adult life. However, VEGF also contributes in several acute and chronic lung disorders [14]. The biological activity of VEGF depends on its reaction with specific receptors. Two high affinity receptors for VEGF have been described, Flt-1 ( $f m s$-like tyrosine kinase 1) and KDR/Flk-1 (fetal liver kinase 1) $[14,15]$.

We hypothesized that the levels of VEGF and receptor Flt-1 would be higher in bronchoalveolar lavage fluid (BALF) from patients with IPF than in patients with pulmonary sarcoidosis. In order to further explore the angiogenetic balance, we measured the mRNA levels of chemokines' receptors, CXCR2 and CXCR3, as well as the biological axis of CXCL12 and cognate receptor, CXCR4.

\section{Patients}

We studied prospectively three groups of patients: (i) 18 patients with IPF, (ii) 16 patients with sarcoidosis, and (iii) 10 normal subjects. Patients' and healthy controls' characteristics are shown in Table 1.

The diagnosis of IPF was made in 8 cases by surgical biopsy (in the correct clinical context, detailed below) and the histologic diagnosis of Usual Interstitial Pneumonia (UIP) was obtained. In the remaining 10 cases the diagnosis was made on the basis of clinical and high-resolution computed tomography (HRCT) criteria: (1) bilateral basal
TABLE 1: Demographic and lung function characteristics of patients with IPF and Pulmonary Sarcoidosis. Values are expressed as mean $\pm \mathrm{SD}$, and age as median (range).

\begin{tabular}{lccc}
\hline Characteristics & Sarcoidosis & IPF & $\begin{array}{c}\text { Normal } \\
\text { subjects }\end{array}$ \\
\hline Number & 16 & 18 & 10 \\
Sex (Male/Female) & $6 / 10$ & $14 / 4$ & $5 / 5$ \\
Age median (yrs) & $53(30-64)$ & $69(56-83)$ & $40(27-62)$ \\
Smokers/ex- & $(3 / 0 / 13)$ & $(10 / 2 / 6)$ & $0 / 0 / 10$ \\
smokers/Nonsmokers & & & \\
FEV (\% pred) & $92.1 \pm 6.7$ & $82.6 \pm 2.6$ & $102 \pm 13$ \\
FVC (\% pred) & $94.7 \pm 6.2$ & $76.2 \pm 2.4$ & $103 \pm 19$ \\
TLC (\% pred) & $87.8 \pm 3.4$ & $65.4 \pm 4.1$ & $95 \pm 4$ \\
DLco (\% pred) & $95.1 \pm 3.1$ & $52.2 \pm 6.1$ & $96 \pm 6$ \\
PaO $($ mmHg) & $80.2 \pm 5.7$ & $71.5 \pm 2.8$ & $85.5 \pm 3.1$ \\
TCC $\times 10^{5} / \mathrm{mL}$ & $30.7 \pm 4.1$ & $24.6 \pm 4.1$ & $20.7 \pm 5.2$ \\
Macrophages\% & $70.1 \pm 8.2$ & $86.1 \pm 2.6$ & $94.2 \pm 1.3$ \\
Neutrophils \% & $3.2 \pm 3.8$ & $6.8 \pm 2.1$ & $3.7 \pm 2.2$ \\
Lymphocytes\% & $23.8 \pm 8.2$ & $5.9 \pm 3.8$ & $1.2 \pm 1.0$ \\
Eosinophils \% & $1.2 \pm 1.9$ & $4.8 \pm 2.8$ & $0.3 \pm 0.4$ \\
\hline
\end{tabular}

TCC: Total cell counts; FVC: Forced Vital Capacity; TLC: Total Lung Capacity; DLCO: Diffusing Capacity for Carbon Monoxide; $\mathrm{P} \alpha \mathrm{O}_{2}$ : Arterial Partial Pressure of Oxygen.

or widespread crackles, (2) restrictive ventilatory defect or isolated depression of $\mathrm{DL}_{\mathrm{CO}},(3)$ computed tomography (CT) appearances indicative of IPF with predominantly basal and subpleural microcystic or macrocystic honeycombing, with variably extensive ground-glass and reticular abnormalities but no consolidation, nodular abnormalities, or other parenchymal abnormalities (apart from centrilobular emphysema), and (4) no environmental exposure to a fibrogenic agent or connective tissue disease [16]. According the aforementioned criteria a known cause of pulmonary fibrosis, such as a connective tissue disorder, has been excluded by both immunologic screening and rheumatological clinical evaluation.

Sarcoidosis diagnosis was made according to the ATS/ERS/World Association of Sarcoidosis and Other Granulomatous Disorders joint statement [4]. All patients had transbronchial or open lung biopsy with histopathological evidence of noncaseating epithelioid cell granulomas without evidence of infection or inorganic material to account for the pulmonary granulomatous reaction. According to chest radiographic classification of sarcoidosis, 3 had stage I disease (lymphadenopathy alone), 7 stage II disease (lymphadenopathy and parenchymal opacities), and 6 stage III disease (only parenchymal opacities).

BALF was also obtained from ten healthy control patients without any past medical history, pulmonary symptoms, or abnormal radiographical findings.

A former smoker was defined as having smoked at least one cigarette per day for 1 year: smoking histories were collected as part of a routine prospective clinical protocol. 
TABLe 2: Primer sequences used for quantitative Real-time RT-PCR.

\begin{tabular}{|c|c|c|c|}
\hline Growth factor or Cytokine & Primer pair Sequence $\left(5^{\prime}-3^{\prime}\right)$ & Annealing temperature & Product size \\
\hline \multirow[t]{2}{*}{ VEGF } & ATGACGAGGGCCTGGAGTGTG & \multirow{2}{*}{$60^{\circ} \mathrm{C}$} & \multirow{2}{*}{91} \\
\hline & CCTATGTGCTGGCCTTGGTGAG & & \\
\hline \multirow[t]{2}{*}{ FLT-1 } & CGGCGGCGGCGAACGAG & \multirow{2}{*}{$58^{\circ} \mathrm{C}$} & \multirow{2}{*}{223} \\
\hline & CATGATGTGCTGGGTGCCTTTTA & & \\
\hline \multirow[t]{2}{*}{ CXCL12 $\alpha$} & TGAGAGCTCGCTTTGAGTGA & \multirow{2}{*}{$55^{\circ} \mathrm{C}$} & \multirow{2}{*}{233} \\
\hline & CACCAGGACCTTCTGTGGAT & & \\
\hline \multirow[t]{2}{*}{ CXCL12 $\beta$} & CTAGTCAAGTGCGTCCACGA & \multirow{2}{*}{$55^{\circ} \mathrm{C}$} & \multirow{2}{*}{221} \\
\hline & GGACACACCACAGCACAAAC & & \\
\hline \multirow[t]{2}{*}{ CXCR2 } & GGCCACTCCAATAACAGCAGGTC & \multirow{2}{*}{$60^{\circ} \mathrm{C}$} & \multirow{2}{*}{197} \\
\hline & GTAGAAAAGGGGGCAGGGTAGAGC & & \\
\hline \multirow[t]{2}{*}{ CXCR3 } & AAAGCAGAGGGGCAGGCAGCACAC & \multirow{2}{*}{$65^{\circ} \mathrm{C}$} & \multirow{2}{*}{181} \\
\hline & AGGGCGGGGAGGTACAGCACGAGT & & \\
\hline \multirow[t]{2}{*}{ CXCR4 } & GGTGGTCTATGTTGGCGTCT & \multirow{2}{*}{$55^{\circ} \mathrm{C}$} & \multirow{2}{*}{229} \\
\hline & TGGAGTGTGACAGCTTGGAG & & \\
\hline
\end{tabular}

TABLE 3: mRNA and protein expression of angiogenetic parameters in IPF and Sarcoidosis. Values are expressed as mean \pm SD.

\begin{tabular}{lccc}
\hline & Sarcoidosis & IPF & $P$ value \\
\hline VEGF-mRNA & $2.99 \pm 1.43$ & $18.3 \pm 14.9$ & .036 \\
VEGF-protein & $154 \pm 36$ & $344 \pm 77$ & $.202(\mathrm{NS})$ \\
(pg/mL) & & & \\
FLT-1-mRNA & $204 \pm 52$ & $154 \pm 33$ & $.434(\mathrm{NS})$ \\
FLT-1protein & $3.0 \pm 1.4$ & $18.8 \pm 6.5$ & .036 \\
$(\mathrm{pg} / \mathrm{mL})$ & & & \\
\hline
\end{tabular}

NS: Nonsignificant, $P<.050$ is considered statistically significant

\section{Methods}

3.1. Pulmonary Function Tests. All patients were evaluated spirometrically and by measurement of lung volumes, diffusion capacity, and arterial blood gases (at rest). Spirometry and lung volumes (helium-dilution technique) and $\mathrm{T}_{L, C O}$ (corrected for the haemoglobin) using the single-breath method were performed by a computerised system (Jaeger 2.12; MasterLab, Würzburg, Germany). Predicted values were obtained from the standardised lung function testing of the European Coal and Steel Community, Luxembourg (1993) [17]. Arterial blood gases were measured by an arterial blood gas analyser (AVL330; MasterLab system).

3.2. BAL Fluid Processing. BALF was obtained from patients with IPF, sarcoidosis, and from normal healthy controls by methods previously described [12, 13]. Briefly, a flexible bronchoscope was wedged into a subsegmental bronchus of a predetermined region of interest based on radiographical findings. A BAL was performed by instilling a total of $240 \mathrm{~mL}$ of normal saline in $60 \mathrm{~mL}$ aliquots, each retrieved by low suction. The BALF fractions were pooled and split equally into two samples. One sample was sent to the clinical microbiology and cytology laboratory and the other sample was placed on ice and transported to the research laboratory. The research sample was filtered through sterile gauze (Thompson, Ontario, Canada) and centrifuged at $400 \mathrm{~g}$ for 15 minutes at $4^{\circ} \mathrm{C}$.

Total cell counts were determined using an improved Neubauer counting chamber and expressed as the total number of cells per $\mathrm{mL}$ of aspirated fluid. The pellet was washed three times with cold PBS-Dulbecco's and the cells were adjusted to a final concentration of $10^{6}$ cells $/ \mathrm{mL}$ with RPMI1640 plus 2\% FCS. The slide preparation was performed as previously reported [18]. The cell-free solution was aliquoted and frozen immediately at $-80^{\circ} \mathrm{C}$ until thawed for chemokine ELISAs.

3.3. Assay of Chemokine Levels Using Specific EnzymeLinked Immunosorbent Assay. Human BALF protein levels of VEGF-A and Flt-1 were quantitated according to the manufacturer's protocol using ELISA kits (R\&D Systems) and measurements were performed in the BALF supernatant. Human VEGF-A and Flt-1 had the lowest detectable limit of 5 and $10 \mathrm{pg} \cdot \mathrm{mL}^{-1}$, respectively.

3.4. RNA Extraction and Reverse Transcription. Total RNA was extracted form each specimen (BALF pellet) using a power homogenizer and the TRIzol reagent (Invitrogen, Carlsband, CA) according to the manufacturer's instructions. cDNA was synthesized using the Strascript reverse transcriptase kit (Stratagene, La Jolla, CA) as previously described [19].

3.5. Real-Time RT-PCR. Peptide growth factors mRNA expression was measured using a real-time RT-PCR assay with SYBR-Green I. Primers were designed to span introns [19]. Glyceraldehyde-3-phosphate dehydrogenase (GAPDH) was used as the internal control, in order to normalize VEGF, flt-1, CXCR2, CXCR3, and both transcripts of CXCL12, CXCL12 $\alpha$ and $\beta$ and CXCR4 expression levels (Table 2). Specifically, $1 \mu \mathrm{L}$ cDNA from pathological or control samples was amplified in a PCR reaction containing $2 \mathrm{X}$ Brilliant SYBR-Green I QPCR Master Mix, $300 \mathrm{nM}$ of each primer 
TABLE 4: mRNA expression of VEGF, FLT-1 and angiogenetic chemokines in healthy subjects (Controls), Sarcoidosis (SARC) and IPF patients. Values are expressed as mean $\pm \mathrm{SD}$.

\begin{tabular}{|c|c|c|c|c|c|c|}
\hline Variables & Controls & IPF & SARC & $P 1$ value & $P 2$ value & $P 3$ value \\
\hline VEGF & $2.82 \pm 3.6$ & $18.3 \pm 14.9$ & $2.99 \pm 1.43$ & NS & .03 & .03 \\
\hline Flt-1 & $134.6 \pm 23.5$ & $154.1 \pm 33.5$ & $204 \pm 52$ & NS & NS & NS \\
\hline CXCR2 & $0.001 \pm 0.002$ & $0.08 \pm 0.25$ & $0.0004 \pm 0.001$ & NS & NS & NS \\
\hline CXCR3 & $0.03 \pm 0.002$ & $0.12 \pm 0.39$ & $0.0001 \pm 0.0004$ & NS & NS & NS \\
\hline CXCR4 & $0.86 \pm 0.64$ & $174.3 \pm 543.6$ & $153.9 \pm 129.3$ & NS & .03 & NS \\
\hline CXCL12 $2 \alpha$ & $92.2 \pm 132.17$ & $168.5 \pm 576.7$ & $1525.2 \pm 2224.7$ & NS & NS & .02 \\
\hline CXCL12 $\beta$ & $223.8 \pm 176.6$ & $142.4 \pm 563.7$ & $822.4 \pm 1423.0$ & NS & NS & NS \\
\hline
\end{tabular}

NS: Non significant, $P<.050$ is considered statistically significant, $P 1: P$ value between controls and IPF,

$P 2: P$ value between controls and Sarcoidosis,

P3: $P$ value between IPF and Sarcoidosis.

and $30 \mu \mathrm{M}$ ROX passive reference dye, in a final volume of $20 \mu \mathrm{L}$. After an initial denaturation at $95^{\circ} \mathrm{C}$ for 10 minutes, the samples were subjected to 40 cycles of amplification, comprised of denaturation at $95^{\circ} \mathrm{C}$ for 30 seconds, annealing at appropriate temperature for each primer pair for 30 seconds and elongation at $72^{\circ} \mathrm{C}$ for 30 seconds, followed by a melt curve analysis, in which the temperature was increased from $55^{\circ} \mathrm{C}$ to $95^{\circ} \mathrm{C}$ at a linear rate of $0.2^{\circ} \mathrm{C} / \mathrm{sec}$. Data collection was performed both during annealing and extension, with two measurements at each step, and at all times during melt curve analysis. In each PCR reaction two nontemplate controls were included. All PCR experiments were conducted on the Mx3000P real-time PCR thermal cycler using the software version 2.00, (Stratagene, La Jolla, CA). To verify the results of the melt curve analysis, PCR products were analyzed by electrophoresis in $2 \%$ agarose gels, stained with ethidium bromide, and photographed on a UV light transilluminator. Primer sequences, annealing temperatures, and PCR products length for all the growth factors analyzed, as well as for GAPDH, are described in Table 2.

All reactions were run in triplicates, and peptide growth factor transcript levels were calculated and normalized to each specimen's house keeping gene mRNA (GAPDH) as well as the appropriate calibrators, using the $\Delta \Delta \mathrm{Ct}$ method for relative quantification. Specifically, after amplification, standard curves were constructed from samples used in a series of consecutive dilutions, for both the gene of interest (GF) and the internal control (GAPDH). Growth factor and GAPDH amplification efficiencies were the same, reaching $100 \%$. IPF and sarcoidosis data were first normalized against variation in sample quality and quantity. Normalized values to GAPDH, $\Delta \mathrm{Cts}$, were initially calculated using the following equation: $\Delta \mathrm{Ct}_{\text {sample }}=\mathrm{Ct}_{\mathrm{GF}}-\mathrm{Ct}_{\mathrm{GAPDH}}$.

\section{Statistical Analysis}

Peptide growth factors mRNA levels were first evaluated by the one-sample Kolmogorov-Smirnov goodness of fit test, in order to determine whether they follow a normal or no distribution. Based on the results, the nonparametric
Spearman test was used to examine correlations. Proportions were compared using chi-square test. The Kruskal-Wallis followed by a posthoc analysis for pairwise significance and the Mann-Whitney $\mathrm{U}$ test were used as indicated to examine growth factors' and chemokines' expression status among IPF and pulmonary sarcoidosis groups. Statistical analysis was carried out using SPSS 13.0 Chicago IL, USA. Statistical significance was set at the $95 \%$ level $(P$-value $<.05)$.

\section{Results}

The demographic and spirometric data of healthy controls, IPF, and sarcoidosis' patients are shown in Table 1. Total cell counts and cell differential were determined and shown in Table 1.

5.1. VEGF Expression in mRNA and Protein Level. A statistically significant increase has been detected in VEGF mRNA expression in IPF in comparison with pulmonary sarcoidosis (mean $\pm \mathrm{SD}, 18.3 \pm 14.9$ versus $2.99 \pm 1.43, P=.03$, resp.) (Table 3, Figure 1). However, no statistically significant difference has been measured in VEGF protein levels between IPF and sarcoidosis patients (mean \pm SD, $344 \pm 77$ versus $154 \pm 36, P=.2$ ), suggesting a posttranscriptional decrease of mRNA expression (Table 3, Figure 2).

A statistically significant increase has been detected in VEGF mRNA expression in sarcoidosis in comparison with healthy subjects (mean $\pm \mathrm{SD}, 2.99 \pm 1.43$ versus $2.82 \pm 3.62$, $P=.03$, resp.). No significant difference has been detected between IPF and control subjects (mean \pm SD, $18.3 \pm 14.9$ versus $2.82 \pm 3.62, P=.4$, resp.) (Table 4 ).

5.2. Flt-1 Expression in mRNA and Protein Level. No statistically significant difference has been measured in Flt-1 mRNA levels between IPF and sarcoidosis patients (mean \pm SD, $154 \pm 33$ versus $204 \pm 52, P=.4$ ) (Table 3, Figure 1). A statistically significant decrease has been detected in Flt1 protein expression in IPF in comparison with pulmonary sarcoidosis (mean $\pm \mathrm{SD}, 18.8 \pm 6.5$ versus $3.0 \pm 1.4, P=.036$, resp.), suggesting a posttranscriptional downregulation of Flt-1 expression (Table 3, Figure 2). 


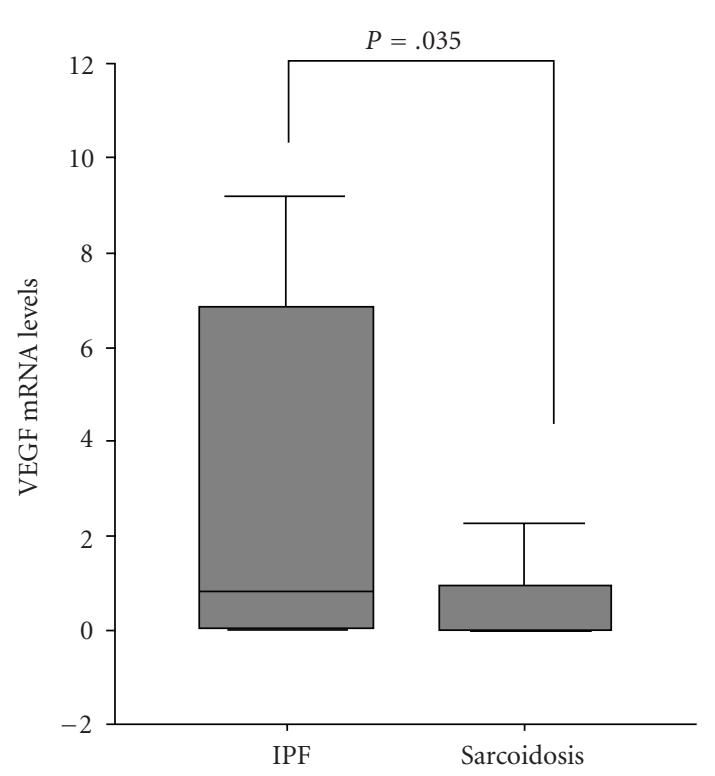

(a)

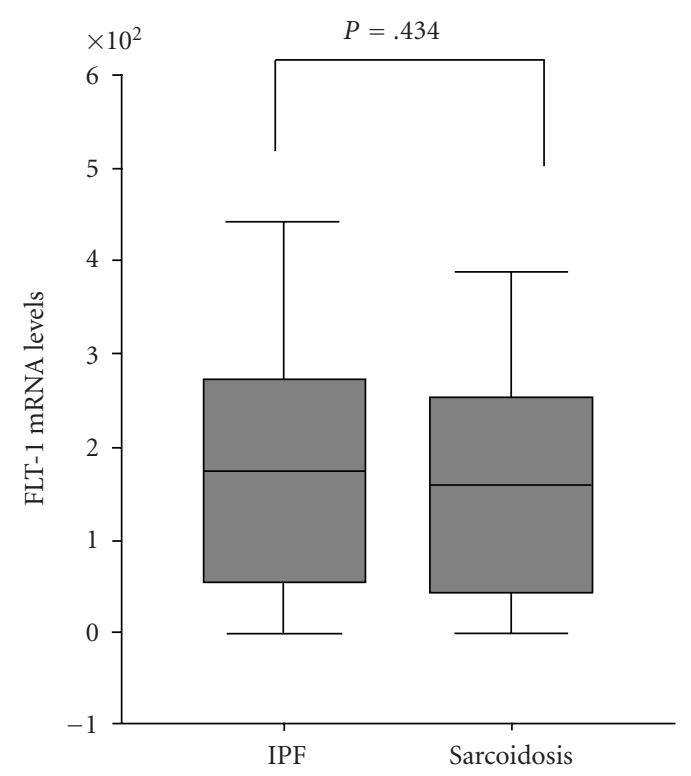

(b)

FIgURE 1: mRNA expression of VEGF/flt-1 in IPF versus Sarcoidosis.

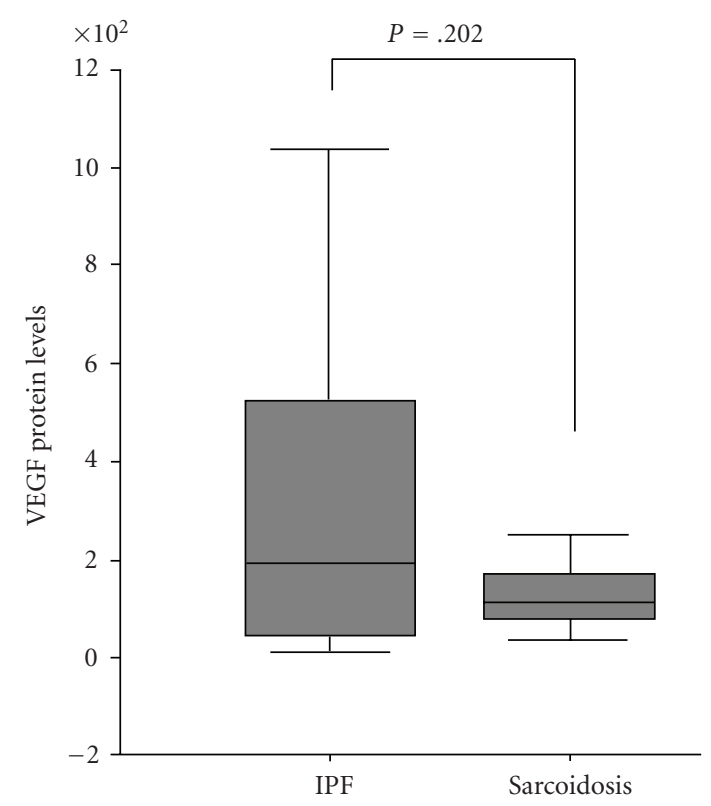

(a)

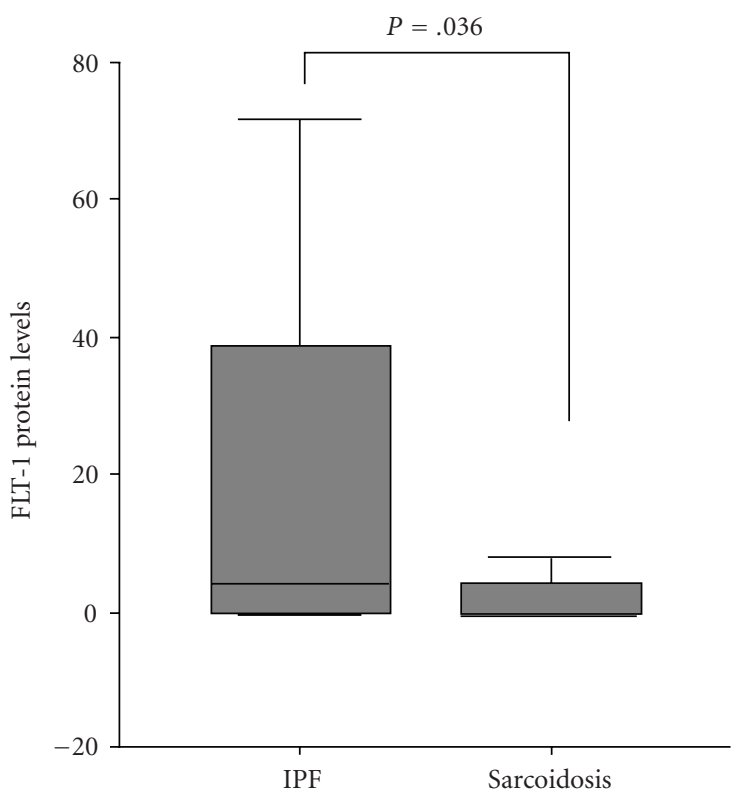

(b)

Figure 2: Protein expression of VEGF/Flt-1 in IPF versus Sarcoidosis.

No significant difference has been measured between healthy controls and sarcoidosis or IPF at flt-1 mRNA expression levels (Table 4).

5.3. Angiogenetic Chemokines (CXCR2, CXCR3, CXCR4, and CXCL12 $\alpha, C X C L 12 \beta)$ mRNA Expression Levels. A significant increase has been measured at CXCL12 $\alpha$ in sarcoidosis' patients in comparison with IPF samples (Table 4). We have also detected that an increase, however, does not reach statistical significance $(P=.06)$ at mRNA levels of CXCR3 in IPF in comparison with sarcoidosis' patients (Table 4).

A statistically significant increase has been detected in CXCR4 mRNA expression in sarcoidosis in comparison with healthy subjects (mean $\pm \mathrm{SD}, 2.40 \pm 1.02$ versus $0.86 \pm 0.64$, $P=.03$, resp.). However, no significant difference has been measured between sarcoidosis and healthy controls at mRNA levels of the other chemokines (Table 4). 
A significant increase has been detected in healthy controls in comparison with IPF at CXCL12 $\beta$ mRNA levels (mean $\pm \mathrm{SD}, 223.8 \pm 176.6$ versus $141.9 \pm 563.2, P=.03$, resp.).

\section{Discussion}

To the best of our knowledge this is the first study to investigate the local expression of the biological axis VEGF and its receptor Flt-1 in patients with IPF and Pulmonary Sarcoidosis without pulmonary fibrosis. Our major finding was an increase of the receptor Flt- 1 at protein level in IPF in comparison with sarcoidosis. Although the increase in VEGF in IPF patients has not been confirmed at the posttranscriptional analysis, this may be due to high variation in the small group of patients analyzed. In order to further investigate the angiogenetic balance between granulomatous and fibrotic disorders, we measured CXC chemokines' receptors and the biological axis of CXCL12/CXCR4. Our major finding is the significant increase in CXCL12 $\alpha$ in sarcoidosis' patients in comparison with IPF.

Recent immunological advances on sarcoidosis have revealed a $\mathrm{T}$ helper 1 (Th1) and $\mathrm{T}$ helper 2 (Th2) paradigm with predominance of the Th1 response in the immunopathogenesis of sarcoidosis $[20,21]$. The concept of disparate activity of the IFN- $\gamma$-induced CXC chemokines in the context of Th1-like immune disorders, such as sarcoidosis, was originally raised by Agostini et al. who documented an enhanced expression of IP-10 in sarcoid tissues and a positive relationship of BALF IP-10 levels and the degree of T-cell alveolitis, suggesting its pivotal role in ruling the migration of T-cells to sites of ongoing inflammation [22]. Our study group has recently showed a shift versus local Th1 immunologic response in sarcoidosis expressed by upregulation of two major Th1cytokines, IL-12 and IL-18 [23-25]. In addition, we have further supported the assertion that IFN- $\gamma$-induced CXC chemokines are strongly involved in the immunomodulatory cascade of sarcoidosis implicating angiostasis with Th1 immune response [12]. However, in the current study, the distinct angiostatic profile expressing by CXCR3 between IPF and sarcoidosis did not reach statistical significance. In addition, Miotto et al. [26] described a specific for Th1-mediated response upregulation of IP-10 BALF levels further implicating angiostatic CXC chemokines in the inflammatory cascade of sarcoidosis. Recently, Katoh et al. reported elevated BALF concentrations of IP-10 and MIG in patients with sarcoidosis and chronic eosinophilic pneumonia [27]. Recent data in a large sarcoid population suggest that CXCL9 and CXCL11 are important mediators in recruiting CXCR3-expressing cells [28]. Importantly, it has been shown that both lymphocytes and cells of monocyte lineage express CXCR3 and are involved in the formation of sarcoid lung granulomas [28].

Furthermore, Sekiya et al. [29] demonstrated a strong correlation of elevated VEGF serum levels with clinical parameters of disease activity in sarcoidosis patients indicating a potential usefulness as a predictor of disease activity and responsiveness to treatment. In contrast BAL fluid VEGF levels from sarcoidosis patients were significantly lower than normal controls as reported by Koyama et al. [30]. Low VEGF levels in the lung environment may reduce angiogenesis and induce apoptosis of vascular endothelial cells thus contributing to the pathogenesis of pulmonary sarcoidosis. It has also been suggested that IP-10 may act as a major chemotactic factor for lymphocytes and ENA78 as a fibrogenic factor, and serum IP-10 levels were more indicative of extrapulmonary lesions [30]. In the current study, we detected an increase of CXCL12 $\alpha$ and its specific receptor, CXCR4, in pulmonary sarcoidosis, in comparison with IPF and controls, respectively, suggesting its major chemotactic role for lymphocytes, in accordance with the aforementioned recent and previous data $[28,31]$.

However, there are several arguments that should be addressed. First of all, we have the small number of patients included in the study; moreover, there is the limitation of several variations regarding the age, disparity of number of males versus females in each group, smoking status, pulmonary function tests, and BALF cell differentiation between the different disease groups and healthy subjects. Secondly, this study is not a morphological one like other elegant reports in lung tissue [32-35]. On the other hand, this is currently difficult, as the use of lung biopsy for the diagnostic approach of sarcoidosis is limited. In addition, it would be interesting to evaluate the angiogenetic process in different sarcoidosis stages.

In line with these findings, our study group demonstrated a distinct local angiogenic profile in patients with IPF compared to sarcoidosis patients. The latter evidence implicates angiogenesis in the fibrotic (Th2) pathway of ILDs and highlights novel noninvasive biomarkers to identify patients who are likely to develop progressive disease allowing anti-inflammatory and other treatments to be evaluated or eventually modified before they have failed. However, further studies are needed in order to better investigate these enigmatic diseases.

\section{Abbreviations}

ACE: Angiotensin Converting Enzyme

BALF: Bronchoalveolar Lavage Fluid

CXCL: CXC ligand

CXCR: CXC receptor

ELISA: Enzyme-linked immunosorbent assay

$\mathrm{FEV}_{1}$ : Forced Expiratory Volume in 1 second

FVC: $\quad$ Forced Vital Capacity

Kco: $\quad$ Carbon monoxide transfer coefficient

IFN- $\gamma$ : Interferon-gamma

IPF: Idiopathic Pulmonary Fibrosis

Th1: $\quad$ T helper 1

VEGF: Vascular Endothelial Growth Factor

UIP: Usual Interstitial Pneumonia

EAA: $\quad$ Extrinsic Allergic Alveolitis

fIIPS: fibrotic Idiopathic Interstitial Pneumonias

fNSIP: fibrotic Nonspecific Interstitial Pneumonia

FLT-1: fms-like tyrosine kinase 1

RT PCR: real time

GAPDH: Glyceraldehyde-3-phosphate dehydrogenase

ATS: American Thoracic Society 
ERS: $\quad$ European Respiratory Society

KDR/Flk-1: fetal liver kinase 1

COP: $\quad$ Cryptogenic Organizing Pneumonia

ILDs: Interstitial Lung Diseases.

\section{References}

[1] M. Turner-Warwick, "Precapillary systemic-pulmonary anastomoses," Thorax, vol. 18, pp. 225-237, 1963.

[2] R. M. Strieter, M. D. Burdick, B. N. Gomperts, et al., "CXC chemokines in angiogenesis," Cytokine \& Growth Factor Reviews, vol. 16, no. 6, pp. 593-609, 2005.

[3] R. M. Strieter, J. A. Belperio, and M. P. Keane, "CXC chemokines in angiogenesis related to pulmonary fibrosis," Chest, vol. 122, no. 6, supplement, pp. 298S-301S, 2002.

[4] G. W. Hunninghake, U. Costabel, M. Ando, et al., "ATS/ERS/ WASOG statement on sarcoidosis. American Thoracic Society/European Respiratory Society/World Association of Sarcoidosis and Granulomatous Disorders," Sarcoidosis Vasculitis and Diffuse Lung Disease, vol. 16, no. 2, pp. 149-173, 1999.

[5] C. Agostini, A. Meneghin, and G. Semenzato, "T-lymphocytes and cytokines in sarcoidosis," Current Opinion in Pulmonary Medicine, vol. 8, no. 5, pp. 435-440, 2002.

[6] M. Petrek, V. Kolek, J. Szotkowská, and R. M. du Bois, "CC and C chemokine expression in pulmonary sarcoidosis," European Respiratory Journal, vol. 20, no. 5, pp. 1206-1212, 2002.

[7] U. Costabel, S. Ohshimo, and J. Guzman, "Diagnosis of sarcoidosis," Current Opinion in Pulmonary Medicine, vol. 14, no. 5, pp. 455-461, 2008.

[8] R. Bonecchi, G. Bianchi, P. P. Bordignon, et al., "Differential expression of chemokine receptors and chemotactic responsiveness of type 1 T helper cells (Th1s) and Th2s," The Journal of Experimental Medicine, vol. 187, no. 1, pp. 129-134, 1998.

[9] A. Capelli, A. Di Stefano, M. Lusuardi, I. Gnemmi, and C. F. Donner, "Increased macrophage inflammatory protein- $1 \alpha$ and macrophage inflammatory protein- $1 \beta$ levels in bronchoalveolar lavage fluid of patients affected by different stages of pulmonary sarcoidosis," American Journal of Respiratory and Critical Care Medicine, vol. 165, no. 2, pp. 236-241, 2002.

[10] K. Katchar, A. Eklund, and J. Grunewald, "Expression of Th1 markers by lung accumulated $\mathrm{T}$ cells in pulmonary sarcoidosis," Journal of Internal Medicine, vol. 254, no. 6, pp. 564-571, 2003.

[11] Y. Nishioka, K. Manabe, J. Kishi, et al., "CXCL9 and 11 in patients with pulmonary sarcoidosis: a role of alveolar macrophages," Clinical and Experimental Immunology, vol. 149, no. 2, pp. 317-326, 2007.

[12] K. M. Antoniou, A. Tzouvelekis, M. G. Alexandrakis, et al., "Different angiogenic activity in pulmonary sarcoidosis and idiopathic pulmonary fibrosis," Chest, vol. 130, no. 4, pp. 982988, 2006.

[13] K. M. Antoniou, N. Tzanakis, E. G. Tzortzaki, et al., "Different angiogenic CXC chemokine levels in bronchoalveolar lavage fluid after interferon $\gamma$-1b therapy in idiopathic pulmonary fibrosis patients," Pulmonary Pharmacology and Therapeutics, vol. 21, no. 6, pp. 840-844, 2008.

[14] N. F. Voelkel, R. W. Vandivier, and R. M. Tuder, "Vascular endothelial growth factor in the lung," American Journal of Physiology, vol. 290, pp. L209-L221, 2006.

[15] B. Millauer, S. Wizigmann-Voos, H. Schnürch, et al., "High affinity VEGF binding and developmental expression suggest Flk-1 as a major regulator of vasculogenesis and angiogenesis," Cell, vol. 72, no. 6, pp. 835-846, 1993.
[16] "American Thoracic Society/European Respiratory Society international multidisciplinary consensus classification of the idiopathic interstitial pneumonias," American Journal of Respiratory and Critical Care Medicine, vol. 165, no. 2, pp. 277304, 2002.

[17] P. H. Quanjer, G. J. Tammeling, J. E. Cotes, et al., "Lung volumes and forced ventilatory flows. Report Working Party Standardization of Lung Function Tests, European Community for Steel and Coal. Official Statement of the European Respiratory Society," The European Respiratory Journal. Supplement, vol. 16, pp. 5-40, 1993.

[18] Report of the European Society of Pneumonology Task Group, "Technical recommendations and guidelines for bronchoalveolar lavage (BAL)," European Respiratory Journal, vol. 2, pp. 561-585, 1989.

[19] N. Soulitzis, I. Karyotis, D. Delakas, and D. A. Spandidos, "Expression analysis of peptide growth factors VEGF, FGF2, TGFB1, EGF and IGF1 in prostate cancer and benign prostatic hyperplasia," International Journal of Oncology, vol. 29, no. 2, pp. 305-314, 2006.

[20] H. Koh, S. Tasaka, N. Hasegawa, et al., "Vascular endothelial growth factor in epithelial lining fluid of patients with acute respiratory distress syndrome," Respirology, vol. 13, no. 2, pp. 281-284, 2008.

[21] J. Muller-Quernheim, "Sarcoidosis: immunopathogenetic concepts and their clinical application," European Respiratory Journal, vol. 12, no. 3, pp. 716-738, 1998.

[22] C. Agostini, M. Cassatella, R. Zambello, et al., "Involvement of the IP-10 chemokine in sarcoid granulomatous reactions," Journal of Immunology, vol. 161, no. 11, pp. 6413-6420, 1998.

[23] I. Tsiligianni, K. M. Antoniou, D. Kyriakou, et al., "Th1/Th2 cytokine pattern in bronchoalveolar lavage fluid and induced sputum in pulmonary sarcoidosis," BMC Pulmonary Medicine, vol. 5, article 8, 2005.

[24] K. M. Antoniou, A. Tzouvelekis, M. G. Alexandrakis, et al., "Upregulation of Th1 cytokine profile (IL-12, IL-18) in bronchoalveolar lavage fluid in patients with pulmonary sarcoidosis," Journal of Interferon and Cytokine Research, vol. 26, no. 6, pp. 400-405, 2006.

[25] K. M. Antoniou, I. Tsiligianni, N. Tzanakis, et al., "Perforin down-regulation and adhesion molecules activation in pulmonary sarcoidosis: an induced sputum and BAL study," Chest, vol. 129, no. 6, pp. 1592-1598, 2006.

[26] D. Miotto, P. Christodoulopoulos, R. Olivenstein, et al., "Expression of IFN- $\gamma$-inducible protein; monocyte chemotactic proteins 1, 3, and 4; and eotaxin in TH1- and TH2-mediated lung diseases," Journal of Allergy and Clinical Immunology, vol. 107, no. 4, pp. 664-670, 2001.

[27] S. Katoh, K. Fukushima, N. Matsumoto, et al., "Accumulation of CXCR3-expressing eosinophils and increased concentration of its ligands (IP10 and Mig) in bronchoalveolar lavage fluid of patients with chronic eosinophilic pneumonia," International Archives of Allergy and Immunology, vol. 137, no. 3, pp. 229235, 2005.

[28] A. Busuttil, S. S. Weigt, M. P. Keane, et al., "CXCR3 ligands are augmented during the pathogenesis of pulmonary sarcoidosis," European Respiratory Journal, vol. 34, no. 3, pp. 676-686, 2009.

[29] M. Sekiya, A. Ohwada, K. Miura, S. Takahashi, and Y. Fukuchi, "Serum vascular endothelial growth factor as a possible prognostic indicator in sarcoidosis," Lung, vol. 181, no. 5, pp. 259-265, 2003.

[30] S. Koyama, E. Sato, M. Haniuda, H. Numanami, S. Nagai, and T. Izumi, "Decreased level of vascular endothelial growth 
factor in bronchoalveolar lavage fluid of normal smokers and patients with pulmonary fibrosis," American Journal of Respiratory and Critical Care Medicine, vol. 166, no. 3, pp. 382385, 2002.

[31] K. Sugiyama, H. Mukae, H. Ishii, et al., "Elevated levels of interferon $\gamma$-inducible protein-10 and epithelial neutrophilactivating peptide-78 in patients with pulmonary sarcoidosis," Respirology, vol. 11, no. 6, pp. 708-714, 2006.

[32] E. A. Renzoni, D. A. Walsh, M. Salmon, et al., "Interstitial vascularity in fibrosing alveolitis," American Journal of Respiratory and Critical Care Medicine, vol. 167, no. 3, pp. 438-443, 2003.

[33] M. P. Keane, "Angiogenesis and pulmonary fibrosis: feast or famine?" American Journal of Respiratory and Critical Care Medicine, vol. 170, pp. 207-209, 2004.

[34] E. A. Renzoni, "Neovascularization in idiopathic pulmonary fibrosis: too much or too little?" American Journal of Respiratory and Critical Care Medicine, vol. 169, pp. 1179-1180, 2004.

[35] M. Ebina, M. Shimizukawa, N. Shibata, et al., "Heterogeneous increase in CD34-positive alveolar capillaries in idiopathic pulmonary fibrosis," American Journal of Respiratory and Critical Care Medicine, vol. 169, no. 11, pp. 1203-1208, 2004. 


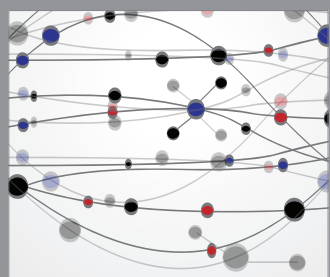

The Scientific World Journal
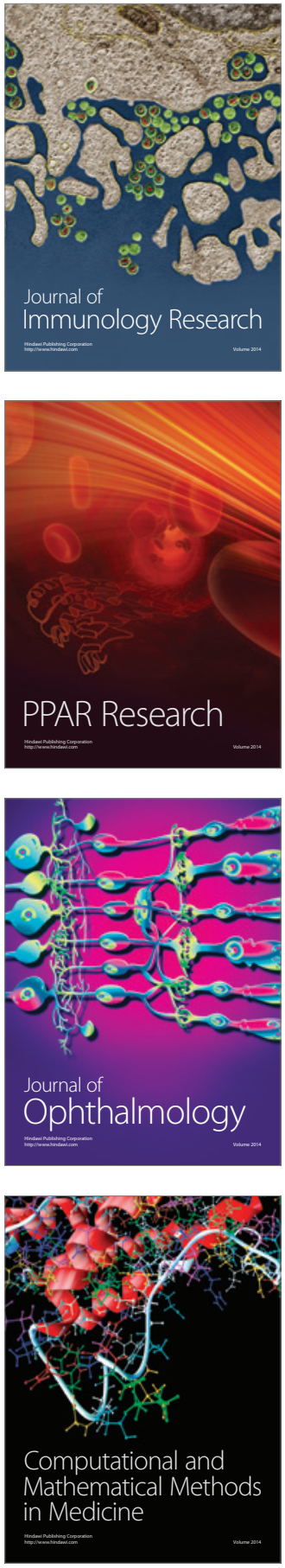

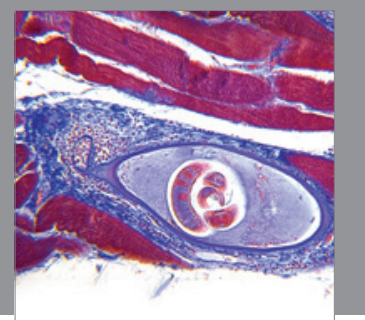

Gastroenterology

Research and Practice
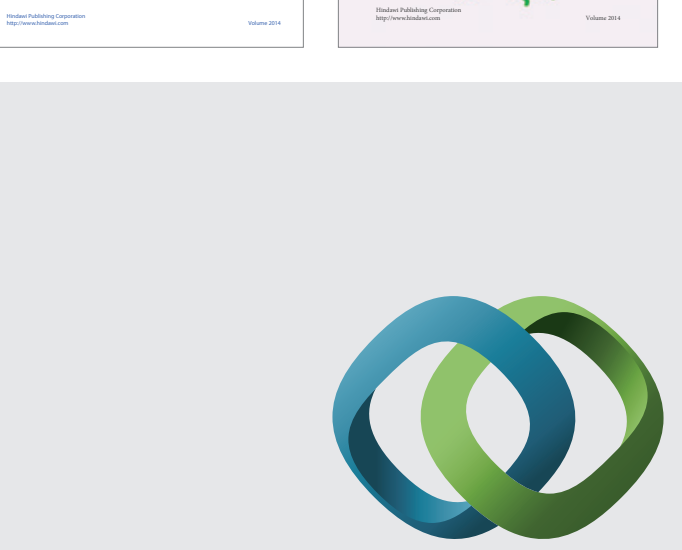

\section{Hindawi}

Submit your manuscripts at

http://www.hindawi.com
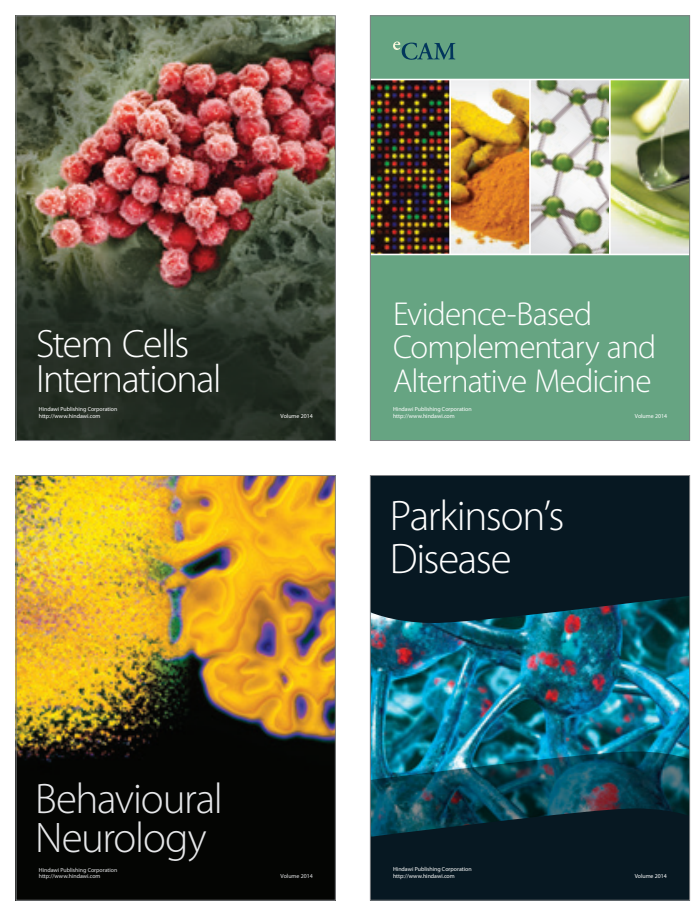

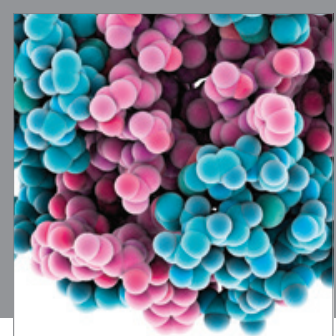

Journal of
Diabetes Research

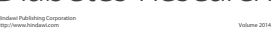

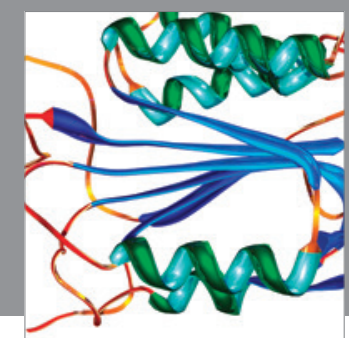

Disease Markers
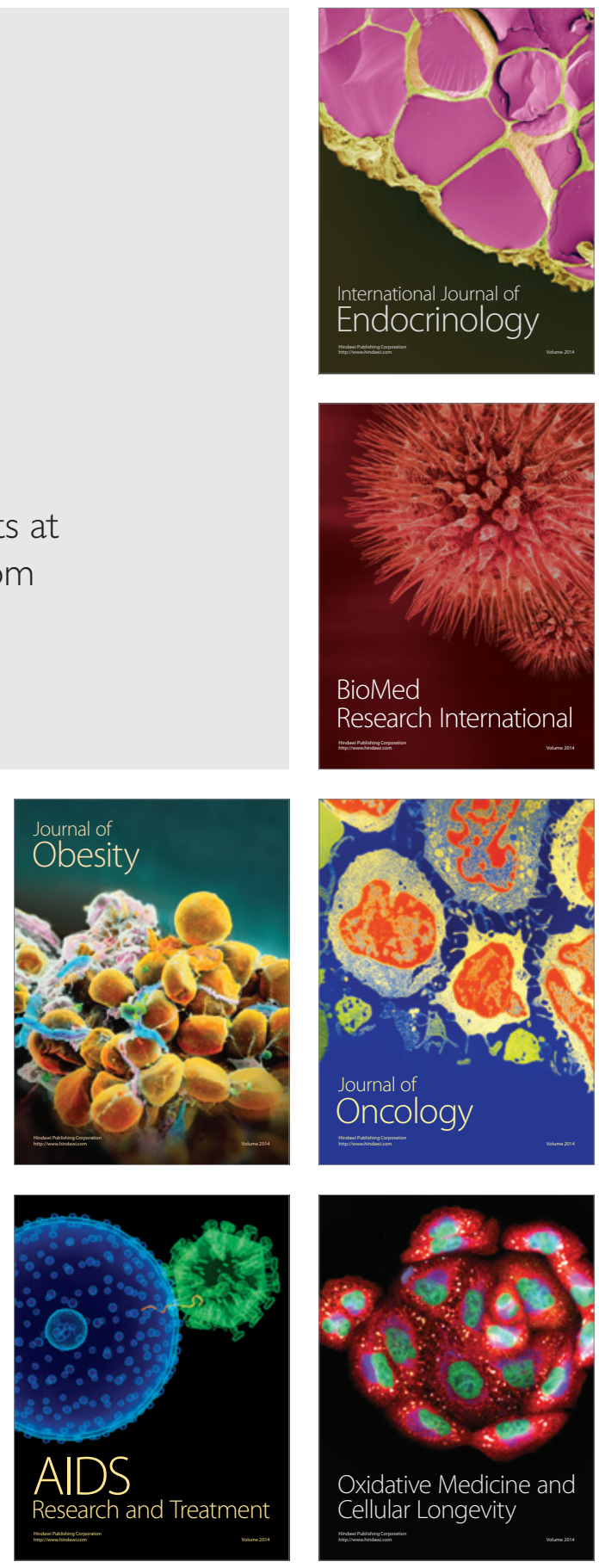